EPJ Web of Conferences 16, 05003 (2011)

DOI: $10.1051 /$ epjconf/20111605003

(C) Owned by the authors, published by EDP Sciences, 2011

\title{
Planet formation in slightly inclined binary systems
}

\author{
J.-W. Xie $1,2, \mathrm{a}$, J.-L. Zhou ${ }^{1}$ and J. Ge ${ }^{2}$ \\ ${ }^{1}$ Department of Astronomy, Nanjing University, Nanjing, Jiangsu 210093, China \\ ${ }^{2}$ Department of Astronomy, University of Florida, Gainesville, FL 32611-2055, USA
}

\begin{abstract}
One of the major problems of planet formation in close binary systems, such as $\alpha$ Centauri $\mathrm{AB}$, is the formation of planetary embryos or cores by mutual accretion of km-sized planetesimals. In this contribution, we test the planetesimal accretion in such close binary systems but with small inclinations $i_{B}=$ $0.1-10^{\circ}$ between the binary orbital plane and the gas disk plane. Compared to previous studies (coplanar case with $i_{B}=0$ ), we find that (1) planetesimal disk is stratified in the vertical direction and planetesimals are redistributed on different orbit groups with respect to their sizes, thus (2) collisions between similarsized bodies dominate, leading to low $\mathrm{dV}$ and favoring planetesimal accretion (3) the planetesimal collision timescale at $1-2 \mathrm{AU}$ is estimated as: $T_{\text {col }}^{B} \sim\left(1+100 i_{B}\right) \times 10^{3}$ yrs, where $0 \leq i_{B} \leq 10^{\circ}$. As a conclusion, although planetesimal accretion are much more favored in slightly inclined binary systems, it is significantly less efficient and slowed-down as compared to the single-star case.
\end{abstract}

\section{INTRODUCTION}

Recent observations show planets can reside in close binary systems with stellar separation of only $20 \mathrm{AU}$. However, the intermediate stage of planet formation, namely the mutual accretion of km-sized planetesimals is strongly affected by the Companion's perturbations [1-4]. Relative velocities $(\triangle V)$ among planetesimals can be pumped up over the threshold velocity, beyond which collision leads to erosion rather than accretion. However, all previous studies have assumed a 2-dimensional disk with a coplanar binary orbit. Extending previous studies by including a 3-dimensional gas disk and an slight inclined binary orbit with tilted angle of $i_{B}=0^{\circ}-10^{\circ}$ with respect to the gas-disk-plane, we numerically investigate the accretion conditions for 1-10 km-sized planetesimals around Alpha Centauri A and B.

\section{KEY RESULTS}

For details, see our recent studies [5, 6]. Here we briefly summarize the key results as following.

1) Planetesimal accretion is possible in slightly inclined binary systems. As shown in Figure 1, for coplanar case $\left(i_{B}=0\right)$, the fraction of accreting collision weighs less than $5 \%$, or still only $\sim 15 \%$ even if including the uncertain ones. This confirms the result of previous studies [2, 3]: planetesimal accretion is suppressed in the $i_{B}=0$ case because high-velocity-collisions between different-sized bodies dominate. On the other hand, for $i_{B}=1^{\circ}$, accretion is much more favored (accreting collision weighs up to $40 \%$ or even $80 \%$ if including the uncertain ones).

2) Planetesimal accretion is much slower in inclined binary systems. Because planetesimals are pumped up to orbits with larger vertical excursion in the inclined case $\left(i_{B}=1^{\circ}\right.$ here) as compared to the singe-star case, their collision rate becomes lower. The planetesimal collision timescale (at 1-2 AU

ae-mail: xiejiwei@gmail.com

This is an Open Access article distributed under the terms of the Creative Commons Attribution-Noncommercial License 3.0, which permits unrestricted use, distribution, and reproduction in any noncommercial medium, provided the original work is properly cited. 

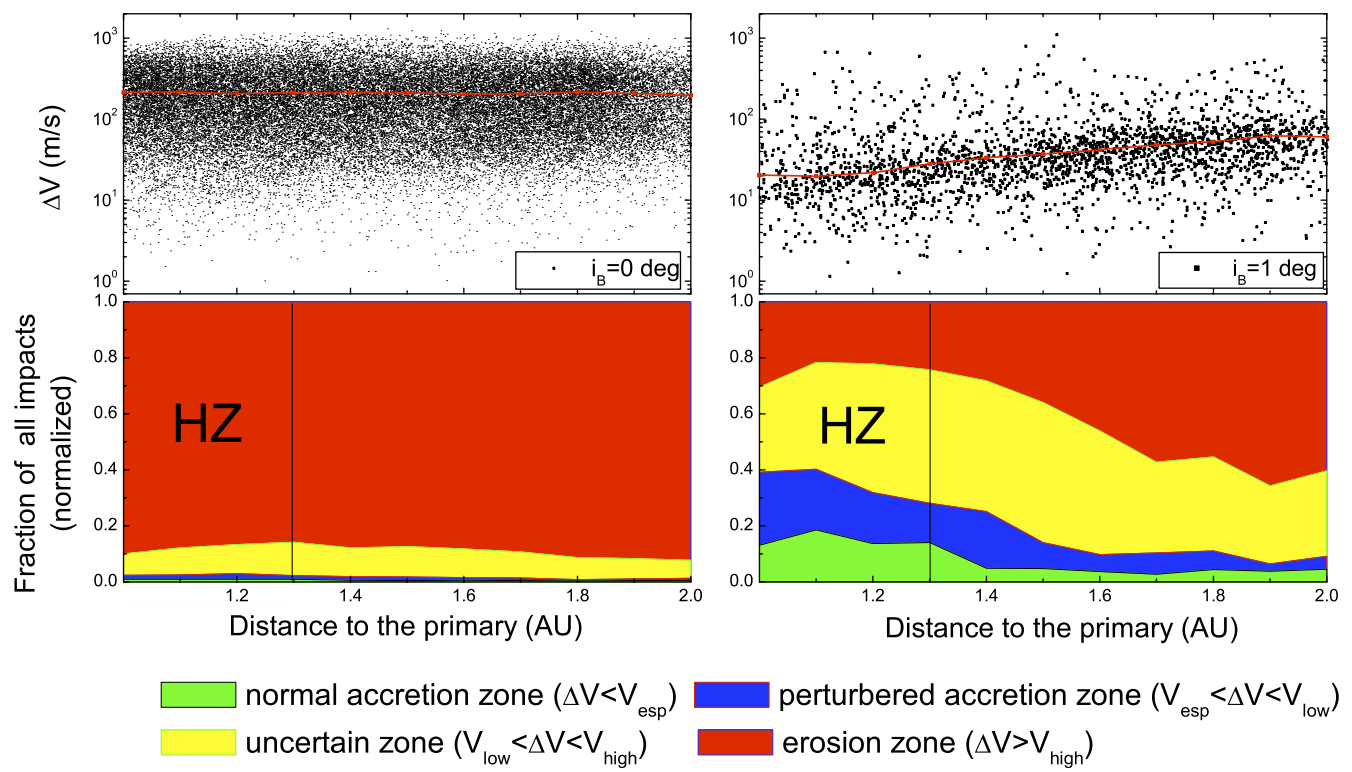

Figure 1. Comparing the accretion conditions for two nominal cases: $i_{B}=0$ and $i_{B}=1 \mathrm{deg}$. The two top panels: distribution of $\triangle V$, as a function of distance to the primary star. Each black point denotes an collision event, and the red scatter-lines are the medium values. The two bottom panels: relative importance of different types of collision outcomes, as a function of distance to the primary star. The vertical lines denote the habitable zone $\sim 1-1.3 \mathrm{AU}$.

to the primary) in a inclined binary system is estimated as:

$$
T_{c o l}^{B} \sim\left(1+100 i_{B}\right) \times 10^{3} \mathrm{yrs},
$$

where $0 \leq i_{B} \leq 10^{\circ}$. Therefore, although planetesimal accretion is much more favored in slightly inclined binary systems, it is significantly less efficient and slowed-down as compared to the singlestar case.

\section{References}

[1] Thébault, P., Marzari, F., \& Scholl, H., Icarus, 183, (2006) 193

[2] Thébault, P., Marzari, F., \& Scholl, H., MNRAS, 388, (2008) 1528

[3] Thébault, P., Marzari, F., \& Scholl, H., MNRAS, 393, (2009) L21

[4] Xie, Ji-Wei, \& Zhou, Ji-Lin, ApJ, 686, (2008) 570

[5] Xie, Ji-Wei, \& Zhou, Ji-Lin, ApJ, 698, (2009) 2066

[6] Xie, Ji-Wei, Zhou, Ji-Lin, \& Ge, Jian, ApJ, to be published 